\title{
Ventricle contact is associated with lower survival and increased peritumoral perfusion in glioblastoma
}

\author{
Bart Roelf Jan van Dijken, BSc, 1 Peter Jan van Laar, MD, PhD,, Chao Li, MD, ${ }^{2,3}$ \\ Jiun-Lin Yan, MD, PhD, ${ }^{2,4,5}$ Natalie Rosella Boonzaier, PhD, ${ }^{2}$ \\ Stephen John Price, MBBS, PhD, FCRS, ${ }^{2}$ and Anouk van der Hoorn, MD, PhD ${ }^{1,2}$
}

\begin{abstract}
1Department of Radiology (EB44), University Medical Center Groningen, University of Groningen, Groningen, The Netherlands; ${ }^{2}$ Cambridge Brain Tumour Imaging Laboratory, Department of Clinical Neurosciences, Division of Neurosurgery, University of Cambridge, Cambridge, United Kingdom; ' ${ }^{2}$ Department of Neurosurgery, Shanghai General Hospital, Shanghai, China; ${ }^{4}$ Department of Neurosurgery, Chang Gung Memorial Hospital, Keelung, Taiwan; and ${ }^{5}$ Department of Neurosurgery, Chang Gung University College of Medicine, Taoyuan, Taiwan
\end{abstract}

OBJECTIVE The purpose of this study was to prospectively investigate outcome and differences in peritumoral MRI characteristics of glioblastomas (GBMs) that were in contact with the ventricles (ventricle-contacting tumors) and those that were not (noncontacting tumors). GBMs are heterogeneous tumors with variable survival. Lower survival is suggested for patients with ventricle-contacting tumors than for those with noncontacting tumors. This might be supported by aggressive peritumoral MRI features. However, differences in MRI characteristics of the peritumoral environment between ventricle-contacting and noncontacting GBMs have not yet been investigated.

METHODS Patients with newly diagnosed GBM underwent preoperative MRI with contrast-enhanced T1-weighted, FLAIR, diffusion-weighted, and perfusion-weighted sequences. Tumors were categorized into ventricle-contacting or noncontacting based on contrast enhancement. Survival analysis was performed using log-rank for univariate analysis and Cox regression for multivariate analysis. Normalized perfusion (relative cerebral blood volume [rCBV]) and diffusion (apparent diffusion coefficient [ADC]) values were calculated in 2 regions: the peritumoral nonenhancing FLAIR region overlapping the subventricular zone and the remaining peritumoral nonenhancing FLAIR region.

RESULTS Overall survival was significantly lower for patients with contacting tumors than for those with noncontacting tumors (434 vs 747 days, $p<0.001$ ). Progression-free survival showed a comparable trend ( 260 vs 375 days, $p=0.094$ ). Multivariate analysis confirmed a survival difference for both overall survival (HR 3.930, 95\% Cl 1.740-8.875, $p=0.001$ ) and progression-free survival (HR 2.506, 95\% Cl 1.254-5.007, $p=0.009$ ). Peritumoral perfusion was higher in contacting than in noncontacting tumors for both FLAIR regions $(p=0.04)$. There was no difference in peritumoral ADC values between the 2 groups.

CONCLUSIONS Patients with ventricle-contacting tumors had poorer outcomes than patients with noncontacting tumors. This disadvantage of ventricle contact might be explained by higher peritumoral perfusion leading to more aggressive behavior.

https://thejns.org/doi/abs/10.3171/2018.5.JNS18340

KEYWORDS glioblastoma; lateral ventricles; survival; magnetic resonance imaging; perfusion; oncology

$\mathrm{G}$ LIOBLASTOMA (GBM) is a poorly understood, very heterogeneous brain tumor. ${ }^{40}$ The outcome for patients is still dismal, with a mean overall survival of 15 months. ${ }^{41}$ Survival, however, varies greatly among GBM patients. Recently, a number of retrospective studies demonstrated that patients with a GBM in contact with the lateral ventricles have poorer survival than patients with tumors that are not in contact with the ventricles (noncontacting tumors).$^{30}$ The mechanism for this poorer survival of patients with ventricle-contacting GBM remains unknown.

A possible explanation could lie within the subven-

ABBREVIATIONS 5-ALA = 5-aminolevulinic acid; $A D C$ = apparent diffusion coefficient; DWI = diffusion-weighted imaging; FLAIR = fluid-attenuated inversion recovery; $\mathrm{FOV}=$ field of view; $\mathrm{GBM}=$ glioblastoma; IDH = isocitrate dehydrogenase; $\mathrm{MGMT}=\mathrm{O}^{6}$-methylguanine-DNA-methyltransferase; $\mathrm{PWI}=$ perfusion-weighted imaging; RANO = Response Assessment in Neuro-Oncology; rCBV = relative cerebral blood volume; SVZ = subventricular zone; TE = echo time; TI = inversion time; TR = repetition time. SUBMITTED February 6, 2018. ACCEPTED May 2, 2018.

INCLUDE WHEN CITING Published online October 19, 2018; DOI: 10.3171/2018.5.JNS18340. 
tricular zone (SVZ), a neurogenic stem cell niche located within the ventricle wall. ${ }^{14}$ Isolation of stem cell-like cells from human GBM samples with similar properties to SVZ stem cells has led to suggestions that the SVZ might initiate gliomagenesis in a subgroup of patients. ${ }^{17,37}$ Previous studies have shown that stem cell-derived GBMs were resistant to current therapy strategies. ${ }^{8,33}$ Ventriclecontacting GBMs could thus demonstrate more aggressive behavior than noncontacting GBMs due to a different cell population. This is further supported by a previous study that observed increasing multifocality and disseminated recurrence among ventricle-contacting GBMs on anatomical imaging. ${ }^{27}$

We hypothesized that such more aggressive behavior of ventricle-contacting tumors due to a stem cell origin could be expected to be visible in the peritumoral environment. Peritumoral infiltration outside the area of contrast enhancement is not resected during surgery and contributes to recurrence. ${ }^{15} \mathrm{~A}$ more aggressive peritumoral environment would show increased relative cerebral blood volume (rCBV) and/or a decreased apparent diffusion coefficient (ADC), representing increased tumor neovascularization and cell density. ${ }^{6}$ However, differences in the peritumoral environment between lateral ventricle-contacting and noncontacting GBM with multimodal MRI have not yet been investigated.

In this study we investigated whether patients with ventricle-contacting GBMs indeed show a lower survival rate than those with noncontacting GBMs in a prospective cohort. Furthermore, we aimed to explain this survival difference by characterizing the peritumoral environment of ventricle-contacting and noncontacting GBMs, with multimodal imaging.

\section{Methods \\ Patient Population}

Patients with a newly diagnosed supratentorial GBM were prospectively recruited for this study from 2010 to 2014. All patients received regular MRI follow-up, and patients with radiologically confirmed recurrence according to the Response Assessment in Neuro-Oncology (RANO) criteria were included ${ }^{45}$ All patients had a World Health Organization performance scale score of $0-1$ and were found appropriate candidates for maximum safe resection by a neurosurgeon with 15 years of experience (S.J.P.). Tumor resection was performed using neuronavigation (StealthStation, Medtronic) and 5-aminolevulinic acid (5ALA) fluorescence guidance. Standard treatment, consisting of concomitant chemoradiotherapy and adjuvant chemotherapy according to the Stupp protocol, was initiated 4 weeks after surgery. ${ }^{41}$ Exclusion criteria were previous cranial surgery, previous cerebral radiotherapy, known other primary tumor, or follow-up outside our hospital (to ensure standardized follow-up). All patients underwent preoperative MRI, preferably 1 day (range 0-9 days) before surgery. Follow-up scans were acquired within 72 hours after surgery, before and after radiotherapy, and at 3-month intervals thereafter. The extent of resection was assessed by measuring pre- and postoperative volumes of the contrastenhancing tumor on contrast-enhanced T1-weighted MRI, conducted in a blinded fashion by a senior researcher and neurosurgeon (J.L.Y.). The extent of resection was considered either gross-total $(100 \%)$ or subtotal $(\geq 78 \%)$ in accordance with previous work. ${ }^{38}$ Progression-free survival, survival after progression, and overall survival were established based on medical records including clinical and imaging information. Progression-free survival was defined according to the RANO criteria. ${ }^{45}$ The tumor samples were investigated for $\mathrm{O}^{6}$-methylguanine-DNA-methyltransferase (MGMT) methylation status using pyrosequencing and isocitrate dehydrogenase (IDH) mutations using immunohistochemistry to the R132H mutation. All tumors were categorized into ventricle-contacting and noncontacting GBMs by a neuroradiologist with 10 years of experience (P.J.V.L.), who was blinded to the patient's history and surgical outcome. Tumors were regarded as ventricle-contacting if the contrast-enhancing lesion touched the lateral ventricles in accordance with previous work. ${ }^{27}$ 5-ALA fluorescence of the ventricle wall was documented during surgery. This study was approved by the local institutional review board, and written informed consent was obtained from all participants. This manuscript was prepared according to the Strengthening the Reporting of Observational Studies in Epidemiology (STROBE) guidelines. ${ }^{44}$

\section{MRI Acquisition}

Preoperative multimodal MRI data acquisition was performed using a 3.0-T Siemens MR Magnetron System (Siemens Healthcare) with a standard 12-channel head coil. Preoperative imaging included 3D T1-weighted contrast-enhanced imaging, a T2-weighted fluid-attenuated inversion recovery (FLAIR) sequence, diffusion-weighted imaging (DWI), and perfusion-weighted imaging (PWI). An anatomical 3D T1-weighted sequence with fat suppression was acquired after the intravenous injection of $9 \mathrm{~mL}$ gadolinium (Gadovist, Bayer Schering Pharma). Imaging parameters for the 3D T1-weighted sequences before and after contrast were as follows: repetition time (TR) 2300 msec, echo time (TE) $2.98 \mathrm{msec}$, inversion time (TI) 900 msec, flip angle $9^{\circ}$, field of view (FOV) $256 \times 240 \mathrm{~mm}$, 176-208 slices, no slice gap, voxel size $1 \times 1 \times 1 \mathrm{~mm}$ ). A 2D FLAIR sequence was also acquired (TR 7840-8420 msec, TE $95 \mathrm{msec}$, TI $2500 \mathrm{msec}$, flip angle $150^{\circ}$, FOV $250 \times 200 \mathrm{~mm}, 25-27$ slices, 1-mm slice gap, voxel size $0.78 \times 0.78 \times 4 \mathrm{~mm}$ ). DWI was acquired with a single-shot echo-planar sequence (TR $8300 \mathrm{msec}$, TE $98 \mathrm{msec}$, flip angle $90^{\circ}$, FOV $192 \times 192 \mathrm{~mm}, 63$ slices, no slice gap, voxel size $2 \times 2 \times 2 \mathrm{~mm})$ using different $\mathrm{b}$ values $(0,350$, $650,1000,1300$, and 1600 seconds $/ \mathrm{mm}^{2}$ ) to generate ADC values. Finally, for PWI a postcontrast dynamic-susceptibility contrast-enhancement sequence was acquired (TR $1500 \mathrm{msec}$, TE $30 \mathrm{msec}$, flip angle $90^{\circ}$, FOV $192 \times 192$ $\mathrm{mm}$, 19 slices, slice gap $1.5 \mathrm{~mm}$, voxel size, $2 \times 2 \times 5 \mathrm{~mm}$ ) with $9 \mathrm{~mL}$ of gadolinium followed by a $20-\mathrm{mL}$ saline flush administered via a power injector at a rate of $5 \mathrm{~mL} / \mathrm{sec}$.

Follow-up imaging was acquired using the same 3.0-T scanner. Follow-up imaging consisted of 3D T1-weighted sequences with fat suppression obtained before and after the intravenous injection of $9 \mathrm{~mL}$ gadolinium (Gadovist, Bayer Schering Pharma); TR $2300 \mathrm{msec}$, TE $2.98 \mathrm{msec}$, TE $900 \mathrm{msec}$, flip angle $9^{\circ}$, FOV $256 \times 240 \mathrm{~mm}, 176-$ 

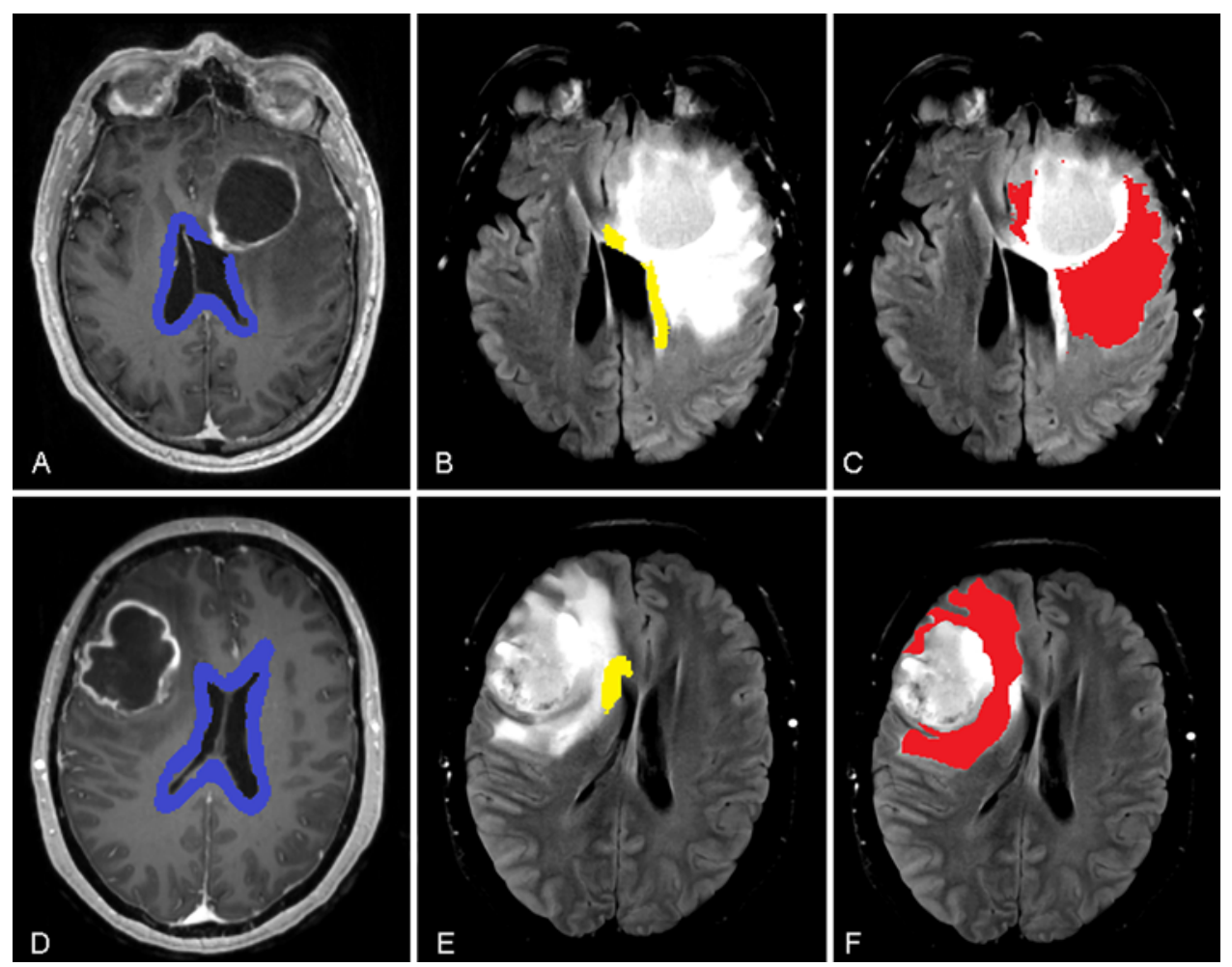

FIG. 1. Regions of interest. Representative MR images obtained in a patient with a ventricle-contacting glioblastoma (upper row) and a patient with a noncontacting glioblastoma (lower row). A and D: Contrast-enhanced T1 weighted images with a 5-mm SVZ surrounding the ventricles (blue). B and E: FLAIR images. The overlap between the high FLAIR signal and the SVZ was included as the first region of interest (yellow). $\mathrm{C}$ and F: The remaining high FLAIR signal outside the T1 contrast-enhancing lesion without SVZ overlap was included as the peritumoral region of interest (red).

208 slices, no slice gap, voxel size $1 \times 1 \times 1 \mathrm{~mm})$ and 2D FLAIR (TR 7840-8420 msec, TE $95 \mathrm{msec}$, TI 2500 msec, flip angle $150^{\circ}$, FOV $250 \times 200 \mathrm{~mm}, 25-27$ slices, 1 -mm slice gap, voxel size $0.78 \times 0.78 \times 4 \mathrm{~mm})$.

\section{Data Processing}

Images were processed using tools from the Oxford Centre for Functional MRI of the Brain (FMRIB) Software Library (FSL) version 5.0.0 (http://fsl.fmrib.ox.ac. uk/fsl/fslwiki/). DWI, PWI, and FLAIR images were coregistered to preoperative contrast-enhanced T1-weighted images by a linear transformation using the FMRIB linear image registration tool (FLIRT) functions provided by FSL. Perfusion data were processed using NordicICE (NordicNeuroLab AS), and maps of rCBV were created following contrast agent leakage correction.

\section{Regions of Interest}

Nonenhancing FLAIR maps were generated from coregistered T2-weighted images in 3D Slicer (http://www. slicer.org). We identified the SVZ as a 5-mm margin surrounding the ventricles corresponding to earlier definitions of the SVZ used by others. ${ }^{9,16,22}$ Ventricle masks were created in GeoS (Springer-Verlag) from the preoperative contrast-enhanced T1-weighted images. Further processing was done in MATLAB (MathWorks Inc.).

Normalized ADC and rCBV values were calculated in 2 regions (see Fig. 1), the nonenhancing peritumoral FLAIR region overlapping with the SVZ (Fig. 1B) and the remaining nonenhancing peritumoral FLAIR region (Fig. 1C). Contralateral normal-appearing white matter control was used to normalize ADC and $\mathrm{rCBV}$ values within the regions.

\section{Statistics}

All collected data were statistically tested in SPSS version 22 (IBM Corp.). Survival analysis was performed for overall survival, progression-free survival, and survival after progression differences between ventricle-contacting and noncontacting tumors with the Kaplan-Meier method, and the survival curves were compared using the log-rank test. Multivariate analysis was performed with Cox regression to determine the influence of various covariates (age, extent of resection, MGMT methylation status, and IDH mutation) on overall survival, progression-free survival, and survival after progression. Comparison of ADC and rCBV values between ventricle-contacting and noncontacting tumors was performed using independent t-tests. Two-sided $\mathrm{p}$ values were used throughout, and an alpha level of 0.05 was used for all analyses.

\section{Results}

\section{General Characteristics}

Out of the 115 initially enrolled patients, a total of 50 
patients were included in this study. The remaining 65 patients were excluded because histology showed a nonGBM tumor $(\mathrm{n}=11)$, radiological data were not accessible ( $\mathrm{n}=11)$, or follow-up was performed outside our hospital $(\mathrm{n}=43)$. The included patients had a median age of 61 years (range 22-69 years), and 70\% were men. All tumors were enhancing on contrast-enhanced T1-weighted imaging. Tumors were contacting the ventricles in 24 of 50 cases $(48 \%)$ and were noncontacting in the remaining 26 (52\%). None of the 26 noncontacting tumors demonstrated 5-ALA fluorescence of the ventricle wall, suggesting that these tumors were true noncontacting lesions. The volume of contrast enhancement was larger for patients with ventricle-contacting tumors $\left(53.5 \pm 27.3 \mathrm{~cm}^{3}\right.$ [mean $\pm \mathrm{SD}$ ] $)$ than those with noncontacting tumors $\left(33.5 \pm 18.7 \mathrm{~cm}^{3}\right)$ $(\mathrm{t}[48]=3.05, \mathrm{p}=0.004)$. However, there was no difference in FLAIR volume between ventricle-contacting tumors $\left(131.1 \pm 61.4 \mathrm{~cm}^{3}\right)$ and noncontacting tumors $(110.5 \pm 60.5$ $\left.\mathrm{cm}^{3}\right)(\mathrm{t}[48]=1.19, \mathrm{p}=0.24)$. Other patient characteristics of the 2 tumor groups were very similar and did not show any statistical significant differences (Table 1).

\section{Survival Analysis}

There was a clear survival difference noticeable between patients with ventricle-contacting and noncontacting tumors. Kaplan-Meier curves for overall survival, progression-free survival, and survival after progression are provided (Fig. 2). Univariate analysis (log-rank) demonstrated that overall survival was significantly different between the 2 patient groups, with patients with ventriclecontacting tumors doing worse (mean $434 \pm 40$ days) than those with noncontacting tumors $(747 \pm 77$ days $),\left(\chi^{2}[1]=\right.$ $11.076, \mathrm{p}<0.001)$. The progression-free survival of patients with ventricle-contacting tumors was shorter than that of those with noncontacting tumors $(260 \pm 33$ days vs 375 \pm 56 days), although this difference was not statistically significant $\left(\chi^{2}[1]=2.802, p=0.09\right)$. Survival after progression was strongly diminished for patients with ventriclecontacting tumors compared to patients with noncontacting tumors $\left(163 \pm 19\right.$ vs $413 \pm 83$ days, $\chi^{2}[1]=11.907, p=$ $0.001)$. Multivariate analysis identified ventricular contact as a prognostic factor in overall survival (HR 3.930, 95\% CI $1.740-8.875, \mathrm{p}=0.001$ ), progression-free survival (HR $2.506,95 \%$ CI $1.254-5.007, p=0.009$ ), and survival after
TABLE 1. General characteristics of patients with ventriclecontacting and noncontacting GBMs

\begin{tabular}{lccc}
\hline \multicolumn{1}{c}{ Characteristic } & $\begin{array}{c}\text { Contacting } \\
(\mathrm{n}=24)\end{array}$ & $\begin{array}{c}\text { Noncontacting } \\
(\mathrm{n}=26)\end{array}$ & $\begin{array}{c}\mathrm{p} \\
\text { Value }\end{array}$ \\
\hline No. of patients & 24 & 26 & \\
\hline Mean age in yrs (range) & $57.2(22.1-68.8)$ & $56.2(36.7-69.2)$ & 0.76 \\
\hline Sex & 17 & & 0.90 \\
$\quad$ Male & 7 & 18 & \\
Female & $53.5 \pm 27.3$ & $33.5 \pm 18.7$ & 0.004 \\
\hline CE volume $\left(\mathrm{cm}^{3}\right)$ & $131.1 \pm 61.4$ & $110.5 \pm 60.5$ & 0.24 \\
\hline FLAIR volume $\left(\mathrm{cm}^{3}\right)$ & & & 0.93 \\
\hline Extent of resection & 17 & 18 & \\
$\quad$ Gross-total & 7 & 8 & \\
Subtotal & & & 0.64 \\
\hline MGMT status & 9 & 11 & \\
Methylated & 15 & 14 & \\
Non-methylated & 0 & 1 & \\
Missing & & & \\
\hline IDH mutation & 2 & 21 & \\
Mutated & 1 & 3 & \\
Wild type & & & \\
Missing & & & \\
\hline
\end{tabular}

$\mathrm{CE}=$ contrast enhancement.

Mean values are presented with SDs.

progression (HR 2.677, 95\% CI 1.170-6.123, $\mathrm{p}=0.02)$ independent of other variables (see Table 2).

\section{Imaging Parameters}

Normalized ADC and rCBV values for ventricle-contacting and noncontacting tumors were calculated in both regions of interest (Table 3). The rCBV was significantly higher for the ventricle-contacting tumors compared to the noncontacting tumors in the peritumoral nonenhancing FLAIR overlapping the SVZ $(\mathrm{t}[39]=2.06, \mathrm{p}=0.04)$. For the remaining peritumoral nonenhancing FLAIR region, ventricle-contacting tumors also showed higher rCBV values than noncontacting tumors $(\mathrm{t}[48]=2.03, \mathrm{p}$ $=0.04)$. Both peritumoral nonenhancing FLAIR regions (i.e., with and without SVZ overlap) showed no difference
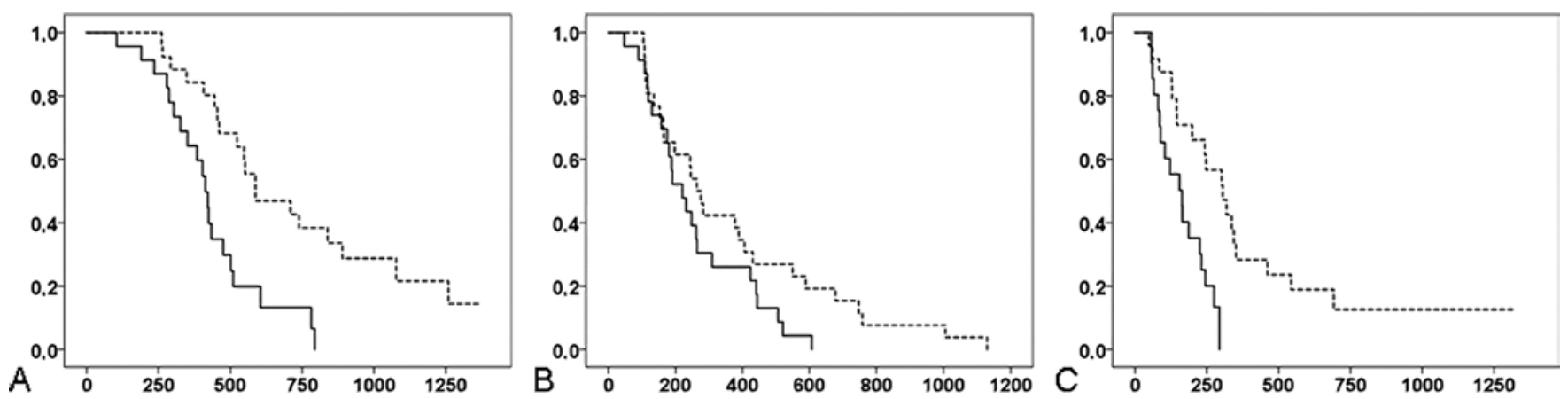

FIG. 2. Kaplan-Meier survival curves for patients with ventricle-contacting and noncontacting GBMs. Survival curves for patients with ventricle-contacting tumors (solid line) and noncontacting tumors (dashed line). A: Overall survival. B: Progression-free survival. C: Survival after progression. Survival in days is displayed on the x-axis, with cumulative survival shown on the y-axis. 
TABLE 2. Multivariate survival analysis

\begin{tabular}{|c|c|c|c|c|c|c|}
\hline \multirow[b]{2}{*}{ Prognostic Factor } & \multicolumn{2}{|c|}{ Overall Survival } & \multicolumn{2}{|c|}{ Progression-Free Survival } & \multicolumn{2}{|c|}{ Survival After Progression } \\
\hline & $\mathrm{HR}(95 \% \mathrm{Cl})$ & $p$ Value & $\mathrm{HR}(95 \% \mathrm{Cl})$ & p Value & $\mathrm{HR}(95 \% \mathrm{Cl})$ & $p$ Value \\
\hline Age & $0.972(0.944-1.000)$ & 0.05 & $1.001(0.976-1.027)$ & 0.93 & $0.994(0.965-1.024)$ & 0.70 \\
\hline Extent of resection & $0.277(0.122-0.631)$ & 0.002 & $0.146(0.063-0.336)$ & $<0.001$ & $0.85(0.346-1.782)$ & 0.56 \\
\hline CE volume & $1.020(1.005-1.035)$ & 0.01 & $0.998(0.983-1.014)$ & 0.83 & $1.024(1.005-1.043)$ & 0.01 \\
\hline FLAIR volume & $0.997(0.990-1.003)$ & 0.32 & $0.995(0.987-1.002)$ & 0.16 & $0.999(0.992-1.006)$ & 0.75 \\
\hline Ventricle contact & $3.930(1.740-8.875)$ & 0.001 & $2.506(1.254-5.007)$ & 0.009 & $2.677(1.170-6.123)$ & 0.02 \\
\hline MGMT methylation & $0.285(0.115-0.706)$ & 0.007 & $0.438(0.222-0.863)$ & 0.01 & $0.700(0.299-1.640)$ & 0.41 \\
\hline IDH mutation & $1.001(1.00-1.002)$ & 0.16 & $1.001(0.999-2.002)$ & 0.25 & $1.000(0.998-1.001)$ & 0.77 \\
\hline
\end{tabular}

in $\mathrm{ADC}(\mathrm{t}[48]=-0.62, \mathrm{p}=0.54$, and $\mathrm{t}[47]=-0.88, \mathrm{p}=$ 0.39 , respectively).

\section{Discussion}

This study provides further support for the earlier suggested survival disadvantage associated with ventricle contact in GBM patients. Patients with tumors contacting the lateral ventricles had a poorer outcome than patients with tumors without ventricle contact, independent of other prognostic factors. Furthermore, rCBV was higher in the peritumoral regions of ventricle-contacting tumors than in the peritumoral regions of noncontacting tumors, while no difference was found for ADC values. Higher $\mathrm{rCBV}$ in the peritumoral region represents aggressive features of tumor cells left behind after surgery. This could explain the survival disadvantage associated with ventricular contact in patients with GBMs.

Our survival data are consistent with previously reported survival rates. ${ }^{7,19,30} \mathrm{~A}$ recently published meta-analysis demonstrated that patients with ventricle-contacting GBMs have both diminished progression-free survival $(-1.8$ months) and diminished overall survival ( -3.5 months), independent of other prognostic factors. ${ }^{30}$ For overall survival, we report a difference of 10 months between ventricle-contacting and noncontacting tumors, corresponding to a very high decrease of $40 \%$ in overall survival. One of the limitations of the aforementioned meta-analysis was the variance in number of confounders among the included studies. Only a small number of studies included the molecular prognostic markers MGMT and IDH in their analyses. ${ }^{2,18,34}$ We included both MGMT methylation and IDH mutation status in our analysis, along with several other possible confounders, and found that ventricle contact was a prognostic factor independent of other factors. In addition to progression-free and overall survival, we also analyzed survival after disease progression. Patients with ventriclecontacting GBMs fared much worse after recurrence. It is possible that ventricle-contacting tumors remain more aggressive throughout initial and second-line treatment due to the presence of therapy-resistant stem cells. ${ }^{8}$ Unfortunately, no details of radiotherapy planning and second-line treatment regimens were available for this study.

In the human brain there are 2 important neural stem cell harboring regions - the subgranular zone and the SVZ, with the latter region being the larger. Stem cell populations that were isolated from GBM samples shared common features with stem cells from the SVZ. ${ }^{17,37}$ Furthermore, in contrast to SVZ contact, subgranular zone involvement has earlier been demonstrated not to alter outcome. ${ }^{29}$ As a result, the SVZ is seen as a possible site of origin in a large subset of GBMs. Lim et al. proposed that only tumors contacting the ventricles should be regarded as stem celloriginating GBMs. ${ }^{27}$ In their study, the authors classified GBMs based on the spatial relation of the contrast-enhancing area to either the SVZ or cortex..$^{27}$ Tumors that contact both the SVZ and the cortex showed the most aggressive nature. It should be noted that these tumors are often larger in size than tumors contacting only one of these regions. ${ }^{27}$ A previous study by Bohman et al. subsequently demonstrated that ventricle-contacting GBMs are larger than noncontacting GBMs. ${ }^{5}$ It remains debatable whether or not the preoperative volume of contrast enhancement is a prognostic factor for survival, as extent of resection is usually seen as a better measure of outcome. . $^{1,311,23,24,28,31}$ In our study we found a limited, yet significant, effect of volume of contrast enhancement on survival. However, the volume of contrast enhancement could not explain the difference between the patient groups, and ventricle contact was identified as a strong independent prognostic factor.

The increased peritumoral rCBV in patients with ventricle-contacting GBMs might well be responsible for the difference in survival between patients with ventricle-contacting and noncontacting tumors. It is well known that the invasive margin of GBMs extends into the nonenhancing FLAIR region. ${ }^{6}$ Increased rCBV has been demonstrated previously in the peritumoral invasive margin of GBM, corresponding with our results. ${ }^{35}$ Such high peritumoral rCBV has been demonstrated to negatively correlate with

TABLE 3. Multisequence MRI parameters in regions of interest for ventricle-contacting and noncontacting GBMs

\begin{tabular}{lccc}
\hline \multicolumn{1}{c}{ Region of Interest } & Contacting & Noncontacting & $p$ Value \\
\hline $\begin{array}{l}\text { Overlap peritumoral FLAIR } \\
\text { w/ SVZ }\end{array}$ & & & \\
\hline rCBV & $2.30 \pm 0.92$ & $1.84 \pm 0.60$ & 0.04 \\
\hline ADC & $1.43 \pm 0.22$ & $1.48 \pm 0.19$ & 0.39 \\
\hline Remaining peritumoral FLAIR & & & \\
\hline rCBV & $1.96 \pm 0.68$ & $1.58 \pm 0.64$ & 0.04 \\
\hline ADC & $1.36 \pm 0.23$ & $1.40 \pm 0.25$ & 0.54 \\
\hline
\end{tabular}


survival. ${ }^{6}$ There is evidence suggesting that stem cells enhance angiogenesis through the expression of vascular endothelial growth factor (VEGF). ${ }^{12,36}$ Increased tumor vascularity resulting from stem cells is also suggested in an animal model with implanted cell cultures comparing cell populations with and without neural stem cell markers. ${ }^{4}$ Stem cells themselves, however, are presumably located in perivascular niches with associated hypoxia. ${ }^{37}$ Hence, stem cells are protected from chemotherapeutic agents, going primarily to areas with high flow. ${ }^{17}$ This can induce further tumor growth and tumor neovascularization. ${ }^{37}$ Other studies also demonstrated low ADC within regions associated with infiltrative disease. ${ }^{6,42}$ Contrary to our hypothesis, we found no difference in ADC values between GBM patients with ventricle-contacting and noncontacting tumors. This might be explained by a lower reliability of DWI for investigating regions in which tumor infiltration is mixed with edema. ${ }^{13}$ A meta-analysis focusing on the diagnostic accuracy of MRI techniques to identify tumor recurrence demonstrated considerably low accuracy for ADC and high performance for perfusion MRI in patients with high-grade gliomas. ${ }^{43}$

The more aggressive peritumoral environment of ventricle-contacting GBMs possibly demands a different treatment approach. ${ }^{26,32}$ Currently, anatomical contrast-enhanced T1-weighted MRI is used for radiotherapy and neurosurgical planning. However, infiltrating tumor cells extend outside the contrast enhancement and are responsible for local recurrent disease. ${ }^{10,15,25}$ There are data suggesting that inclusion of the SVZ in radiotherapy planning has a positive effect on outcome., ${ }^{9,16,22}$ Furthermore, the increased peritumoral perfusion of ventricle-contacting GBMs could be targeted with neurosurgery. Using rCBV maps in neurosurgical planning of ventricle-contacting GBMs could possibly change the extent of resection, thereby improving outcome for patients with these tumors. We encourage future studies to further investigate inclusion of rCBV maps in neurosurgical planning.

Our study has several limitations. First, our sample size is relatively small. Although the number of patients in the 2 tumor groups was limited, it was large enough to demonstrate a statistically significant difference in survival and peritumoral perfusion between the groups. Second, it is not known whether increased rCBV values are a component of normal periventricular anatomy. However, the rCBV values were higher in both the periventricular and the remaining FLAIR regions in patients with ventricle-contacting tumors than in those with noncontacting tumors, which cannot be explained by periventricular anatomy. Third, there were no tissue samples available for histological correlations with our imaging results. However, previous studies have clearly showed a correlation between increased rCBV values and worse outcome. ${ }^{6,20,21,39}$

\section{Conclusions}

We prospectively showed that GBM patients with tumors contacting the ventricles have significantly worse survival. The peritumoral FLAIR regions of ventricle-contacting GBMs were suggestive of a more aggressive environment demonstrated by higher $\mathrm{CCBV}$ values than the peritumoral environment of noncontacting GBMs. Our results provide arguments to include rCBV maps in neurosurgical planning for the treatment of ventricle-contacting GBMs.

\section{Acknowledgments}

This study was partially funded by a National Institutes of Health clinician scientist fellowship to S. J. Price.

\section{References}

1. Abdullah KG, Ramayya A, Thawani JP, Macyszyn L, Martinez-Lage M, O'Rourke DM, et al: Factors associated with increased survival after surgical resection of glioblastoma in octogenarians. PLoS One 10:e0127202, 2015

2. Adeberg S, König L, Bostel T, Harrabi S, Welzel T, Debus J, et al: Glioblastoma recurrence patterns after radiation therapy with regard to the subventricular zone. Int J Radiat Oncol Biol Phys 90:886-893, 2014

3. Babu R, Komisarow JM, Agarwal VJ, Rahimpour S, Iyer A, Britt D, et al: Glioblastoma in the elderly: the effect of aggressive and modern therapies on survival. J Neurosurg 124:998-1007, 2016

4. Bao S, Wu Q, Sathornsumetee S, Hao Y, Li Z, Hjelmeland $\mathrm{AB}$, et al: Stem cell-like glioma cells promote tumor angiogenesis through vascular endothelial growth factor. Cancer Res 66:7843-7848, 2006

5. Bohman LE, Swanson KR, Moore JL, Rockne R, Mandigo C, Hankinson T, et al: Magnetic resonance imaging characteristics of glioblastoma multiforme: implications for understanding glioma ontogeny. Neurosurgery 67:1319-1328, 2010

6. Boonzaier NR, Larkin TJ, Matys T, van der Hoorn A, Yan JL, Price SJ: Multiparametric MR imaging of diffusion and perfusion in contrast-enhancing and nonenhancing components in patients with glioblastoma. Radiology 284:180-190, 2017

7. Chaichana K, Parker S, Olivi A, Quiñones-Hinojosa A: A proposed classification system that projects outcomes based on preoperative variables for adult patients with glioblastoma multiforme. J Neurosurg 112:997-1004, 2010

8. Chen J, Li Y, Yu TS, McKay RM, Burns DK, Kernie SG, et al: A restricted cell population propagates glioblastoma growth after chemotherapy. Nature 488:522-526, 2012

9. Chen L, Guerrero-Cazares H, Ye X, Ford E, McNutt T, Kleinberg L, et al: Increased subventricular zone radiation dose correlates with survival in glioblastoma patients after gross total resection. Int J Radiat Oncol Biol Phys 86:616622, 2013

10. Cordova JS, Shu HKG, Liang Z, Gurbani SS, Cooper LAD, Holder CA, et al: Whole-brain spectroscopic MRI biomarkers identify infiltrating margins in glioblastoma patients. Neuro Oncol 18:1180-1189, 2016

11. Crawford FW, Khayal IS, McGue C, Saraswathy S, Pirzkall A, Cha S, et al: Relationship of pre-surgery metabolic and physiological MR imaging parameters to survival for patients with untreated GBM. J Neurooncol 91:337-351, 2009

12. D'Alessio A, Proietti G, Lama G, Biamonte F, Lauriola L, Moscato U, et al: Analysis of angiogenesis related factors in glioblastoma, peritumoral tissue and their derived cancer stem cells. Oncotarget 7:78541-78556, 2016

13. Dhermain FG, Hau P, Lanfermann H, Jacobs AH, van den Bent MJ: Advanced MRI and PET imaging for assessment of treatment response in patients with gliomas. Lancet Neurol 9:906-920, 2010

14. Doetsch F, Caillé I, Lim DA, García-Verdugo JM, AlvarezBuylla A: Subventricular zone astrocytes are neural stem cells in the adult mammalian brain. Cell 97:703-716, 1999

15. Eidel O, Burth S, Neumann JO, Kieslich PJ, Sahm F, Jungk $\mathrm{C}$, et al: Tumor infiltration in enhancing and non-enhancing parts of glioblastoma: a correlation with histopathology. PLoS One 12:e0169292, 2017

16. Evers P, Lee PP, DeMarco J, Agazaryan N, Sayre JW, Selch $\mathrm{M}$, et al: Irradiation of the potential cancer stem cell niches 
in the adult brain improves progression-free survival of patients with malignant glioma. BMC Cancer 10:384, 2010

17. Galli R, Binda E, Orfanelli U, Cipelletti B, Gritti A, De Vitis $\mathrm{S}$, et al: Isolation and characterization of tumorigenic, stemlike neural precursors from human glioblastoma. Cancer Res 64:7011-7021, 2004

18. Han S, Li X, Qiu B, Jiang T, Wu A: Can lateral ventricle contact predict the ontogeny and prognosis of glioblastoma? J Neurooncol 124:45-55, 2015

19. Jafri NF, Clarke JL, Weinberg V, Barani IJ, Cha S: Relationship of glioblastoma multiforme to the subventricular zone is associated with survival. Neuro Oncol 15:91-96, 2013

20. Jain R, Poisson L, Narang J, Gutman D, Scarpace L, Hwang $\mathrm{SN}$, et al: Genomic mapping and survival prediction in glioblastoma: molecular subclassification strengthened by hemodynamic imaging biomarkers. Radiology 267:212-220, 2013

21. Jain R, Poisson LM, Gutman D, Scarpace L, Hwang SN, Holder CA, et al: Outcome prediction in patients with glioblastoma by using imaging, clinical, and genomic biomarkers: focus on the nonenhancing component of the tumor. Radiology 272:484-493, 2014

22. Khalifa J, Tensaouti F, Lusque A, Plas B, Lotterie JA, Benouaich-Amiel A, et al: Subventricular zones: new key targets for glioblastoma treatment. Radiat Oncol 12:67, 2017

23. Kuhnt D, Becker A, Ganslandt O, Bauer M, Buchfelder M, Nimsky C: Correlation of the extent of tumor volume resection and patient survival in surgery of glioblastoma multiforme with high-field intraoperative MRI guidance. Neuro Oncol 13:1339-1348, 2011

24. Lacroix M, Abi-Said D, Fourney DR, Gokaslan ZL, Shi W, DeMonte F, et al: A multivariate analysis of 416 patients with glioblastoma multiforme: prognosis, extent of resection, and survival. J Neurosurg 95:190-198, 2001

25. Lemée JM, Clavreul A, Menei P: Intratumoral heterogeneity in glioblastoma: don't forget the peritumoral brain zone. Neuro Oncol 17:1322-1332, 2015

26. Liang HKT, Chen WY, Lai SF, Su MY, You SL, Chen LH, et al: The extent of edema and tumor synchronous invasion into the subventricular zone and corpus callosum classify outcomes and radiation therapy strategies of glioblastomas. Int J Radiat Oncol Biol Phys 99:S187-S188, 2017

27. Lim DA, Cha S, Mayo MC, Chen MH, Keles E, VandenBerg $S$, et al: Relationship of glioblastoma multiforme to neural stem cell regions predicts invasive and multifocal tumor phenotype. Neuro Oncol 9:424-429, 2007

28. Mazaris P, Hong X, Altshuler D, Schultz L, Poisson LM, Jain $\mathrm{R}$, et al: Key determinants of short-term and long-term glioblastoma survival: a 14-year retrospective study of patients from the Hermelin Brain Tumor Center at Henry Ford Hospital. Clin Neurol Neurosurg 120:103-112, 2014

29. Mistry AM, Dewan MC, White-Dzuro GA, Brinson PR, Weaver KD, Thompson RC, et al: Decreased survival in glioblastomas is specific to contact with the ventricular-subventricular zone, not subgranular zone or corpus callosum. J Neurooncol 132:341-349, 2017

30. Mistry AM, Hale AT, Chambless LB, Weaver KD, Thompson $\mathrm{RC}$, Ihrie RA: Influence of glioblastoma contact with the lateral ventricle on survival: a meta-analysis. J Neurooncol 131:125-133, 2017

31. Nestler U, Lutz K, Pichlmeier U, Stummer W, Franz K, Reulen HJ, et al: Anatomic features of glioblastoma and their potential impact on survival. Acta Neurochir (Wien) 157:179-186, 2015

32. Nourallah B, Digpal R, Jena R, Watts C: Irradiating the subventricular zone in glioblastoma patients: is there a case for a clinical trial? Clin Oncol (R Coll Radiol) 29:26-33, 2017

33. Piccirillo SGM, Spiteri I, Sottoriva A, Touloumis A, Ber S, Price SJ, et al: Contributions to drug resistance in glioblastoma derived from malignant cells in the sub-ependymal zone. Cancer Res 75:194-202, 2015
34. Pina Batista KM, Vega IF, de Eulate-Beramendi SA, Morales J, Kurbanov A, Asnel D, et al: Prognostic significance of the markers IDH1 and YKL40 related to the subventricular zone. Folia Neuropathol 53:52-59, 2015

35. Price SJ, Young AMH, Scotton WJ, Ching J, Mohsen LA, Boonzaier NR, et al: Multimodal MRI can identify perfusion and metabolic changes in the invasive margin of glioblastomas. J Magn Reson Imaging 43:487-494, 2016

36. Roos A, Ding Z, Loftus JC, Tran NL: Molecular and microenvironmental determinants of glioma stem-like cell survival and invasion. Front Oncol 7:120, 2017

37. Sanai N, Alvarez-Buylla A, Berger MS: Neural stem cells and the origin of gliomas. N Engl J Med 353:811-822, 2005

38. Sanai N, Polley MY, McDermott MW, Parsa AT, Berger MS: An extent of resection threshold for newly diagnosed glioblastomas. J Neurosurg 115:3-8, 2011

39. Schmainda KM, Zhang Z, Prah M, Snyder BS, Gilbert MR, Sorensen AG, et al: Dynamic susceptibility contrast MRI measures of relative cerebral blood volume as a prognostic marker for overall survival in recurrent glioblastoma: results from the ACRIN 6677/RTOG 0625 multicenter trial. Neuro Oncol 17:1148-1156, 2015

40. Sottoriva A, Spiteri I, Piccirillo SGM, Touloumis A, Collins VP, Marioni JC, et al: Intratumor heterogeneity in human glioblastoma reflects cancer evolutionary dynamics. Proc Natl Acad Sci U S A 110:4009-4014, 2013

41. Stupp R, Mason WP, van den Bent MJ, Weller M, Fisher B, Taphoorn MJB, et al: Radiotherapy plus concomitant and adjuvant temozolomide for glioblastoma. $\mathbf{N}$ Engl J Med 352:987-996, 2005

42. van der Hoorn A, Yan JL, Larkin TJ, Boonzaier NR, Matys T, Price SJ: Posttreatment apparent diffusion coefficient changes in the periresectional area in patients with glioblastoma. World Neurosurg 92:159-165, 2016

43. van Dijken BRJ, van Laar PJ, Holtman GA, van der Hoorn A: Diagnostic accuracy of magnetic resonance imaging techniques for treatment response evaluation in patients with high-grade glioma, a systematic review and meta-analysis. Eur Radiol 27:4129-4144, 2017

44. von Elm E, Altman DG, Egger M, Pocock SJ, Gøtzsche PC, Vandenbroucke JP: The Strengthening the Reporting of Observational Studies in Epidemiology (STROBE) statement: guidelines for reporting observational studies. J Clin Epidemiol 61:344-349, 2008

45. Wen PY, Macdonald DR, Reardon DA, Cloughesy TF, Sorensen AG, Galanis E, et al: Updated response assessment criteria for high-grade gliomas: response assessment in neurooncology working group. J Clin Oncol 28:1963-1972, 2010

\section{Disclosures}

The authors report no conflict of interest concerning the materials or methods used in this study or the findings specified in this paper.

\section{Author Contributions}

Conception and design: all authors. Acquisition of data: van Dijken, van Laar, Li, Yan, Price, van der Hoorn. Analysis and interpretation of data: van Dijken, Li, Yan, Boonzaier, Price, van der Hoorn. Drafting the article: all authors. Critically revising the article: all authors. Reviewed submitted version of manuscript: van Laar, Li, Yan, Boonzaier, Price, van der Hoorn. Approved the final version of the manuscript on behalf of all authors: van Dijken. Statistical analysis: van Dijken. Study supervision: van Laar, Price, van der Hoorn.

\section{Correspondence}

Bart Roelf Jan van Dijken: University Medical Center Groningen, The Netherlands.b.r.j.van.dijken@umcg.nl. 